\title{
Kojic acid derivatives as potential anticancer agents: Synthesis and cytotoxic evaluation on A375 human malignant melanoma cells
}

\author{
Gülşah KARAKAYA ${ }^{1} \mathbb{D}$, Ayşe ERCAN ${ }^{2}$ (D) , Selin ÖNCÜL ${ }^{2}$ (D) , Mutlu Dilsiz AYTEMİR ${ }^{1 * \mathbb{D}}$ \\ 1 Department of Pharmaceutical Chemistry, Faculty of Pharmacy, Hacettepe University, Sihhiye 06100 Ankara, \\ Turkey. \\ 2 Department of Biochemistry, Faculty of Pharmacy, Hacettepe University, S1hhiye 06100 Ankara, Turkey. \\ * Corresponding Author. E-mail: mutlud@hacettepe.edu.tr (M.D.A.); Tel. + 903123240333 - 114.
}

Received: 18 February 2019 / Revised: 28 May 2019 / Accepted: 29 May 2019

ABSTRACT: Malignant melanoma is a serious type of skin cancer with high mortality rates, arising from melanocytic cells responsible for the pigmentation of the skin. Besides, the excessive accumulation of melanin pigment can lead to many other hyperpigmentation disorders. Kojic acid is used as a skin lightening agent of medicinal and cosmetic products used in hyperpigmentation and sunburn cases. In the present study, substituted halogen containing benzylpiperazine derivatives of kojic acid (compounds 1-9) were synthesized via Mannich reaction in mild conditions. Afterwards, the cyclic amine derivatives (compounds 10-26) were obtained as a result of nucleophilic substitutions. Cytotoxic effects of all the compounds on A375 human malignant melanoma cell lines were explored by sulphorhodamine B assay and efficacies has been compared to those of anticancer FDA-approved drugs dacarbazine, temozolomide, and lenalidomide, currently used in the treatment of malignant melanoma. Also, the most active two compounds were also tested on healthy cell lines (HGF-1 and MRC-5 cell lines). These compounds have shown highest activity ( $\mathrm{IC}_{50}$ : 71.27 for compound 9 and $73.74 \mu \mathrm{M}$ for compound $\mathbf{1}$ ) than temozolomide $\left(\mathrm{IC}_{50}: 95.6 \mu \mathrm{M}\right)$ and lenalidomide $\left(\mathrm{IC}_{50}: 143.1 \mu \mathrm{M}\right)$ against $\mathrm{A} 375$ cells though giving less harm to non-cancerous cell lines. In conclusion, these compounds stand out as promising anticancer agents for further studies.

KEYWORDS: Kojic acid; synthesis; A375 cells; Mannich bases.

\section{INTRODUCTION}

Melanin is a biopolymer that contributes to pigmentation of skin and hair in mammals. Under normal physiological conditions, it provides the protection of skin against hazardous ultraviolet radiation and prevents cancer development. However, abnormal accumulation of melanin is associated with various skin hyperpigmentation disorders ranging from chloasma, melasma, senile lentigines, melanoderma to malignant melanoma [1]. Malignant melanoma is the leading cause of death from skin cancer. Although it is the least common form of skin cancer, it has the highest mortality rate [2]. The median overall survival in the treatment of malignant melanoma is around 3 months demonstrating the useless of chemotherapy regimens including dacarbazine, approved agent, which have response rates between $6 \%$ and $15 \%$ [3]. Also, ipilimumab and vemurafenib are approved by European Medicines Agency (EMA) whereas oncolytic virus therapy with the genetically engineered herpes simplex virus was also approved by the Food and Drug Administration (FDA) in 2015 [4-6]. Besides, dermatological problems are serious esthetic problems in human beings having a strong impact on the patients's emotional state and quality of life. For the stated reasons treatment of hiperpigmentation is an attractive target for the development of new depigmenting agents with further application in pharmacological and cosmetic fields. Currently, the safest and the most effective way to treat cutaneous hyperpigmentation is said to be reducing melanin production by inhibiting tyrosinase activity which is the critical rate-limiting enzyme [7].

Kojic acid (5-hydroxy-2-(hydroxymethyl)- $4 \mathrm{H}$-pyran-4-one, KA) is a fungal metabolite produced by many species of Aspergillus niger and Penicillium and known with its tyrosinase inhibitor activity which is the critical rate-limiting enzyme in melanin synthesis. This depigmenting effect of KA is attributed to its ability to chelate copper at the active site of the enzyme [8]. By virtue of this function, it is widely used as a skin

How to cite this article: Karakaya G, Ercan A, Öncül S, Aytemir MD. Kojic acid derivatives as potent anticancer agents: Synthesis and cytotoxic evaluation on A375 human malignant melanoma cells. J Res Pharm. 2019; 23(4): 596-607. 
lightening or bleaching agent in cosmetic products and as a food additive for preventing enzymatic discoloration [9].

Cellular internalization of hydrophilic molecules is still a compelling problem because of the nature of plasma membrane. So therapeutic agents should have enough lipophilicity to be delivered at the targeted sites. In order to circumvent this impermeability of the barrier, Mannich bases are prepared commonly in organic chemistry. Moreover, Mannich bases having anti-carcinogenic activity are reported that they didn't alkylate cellular hydroxyl and amino groups causing mutagenicity and genotoxicity [10]. To date, a large number of Mannich bases of KA were synthesized by our research group and their various biological actions including anticonvulsant, antibacterial, antifungal, anti-mycobacterial, anti-aging, antioxidant, antityrosinase and antiviral activities with cytotoxicity were evaluated [11-18]. In our recent study, the synthesis of new KA derivatives and their inhibitory activities on melanogenesis were reported [19]. Some compounds were found to be significantly more potent than the FDA-approved drugs dacarbazine, temozolomide, and lenalidomide which were used as standards. More importantly, most of these novel compounds desirably represented no harm to healthy cell lines while having killed cancerogenic cell lines. Promising results were submitted to Turkish and International Patent (TR2017/20155 and PCT/TR2018/050724).

In the light of these discoveries, herein twenty six Mannich bases comprising eleven new compounds, in the structure of 2-substituted-3-hydroxy-6-hyroxymethyl/chloromethyl/morpholinylmethyl/ piperidinylmethyl/pyrrolidinylmethyl-4H-pyran-4-one were synthesized and evaluated for their cytotoxic effects on A375 human melanoma cells by using sulphorhodamine B (SRB) assay. Dacarbazine, temozolomide and lenalidomide which are currently used agents in the treatment of malignant melanoma, were used as control agents. The cytotoxicity against healthy cells HGF-1 (human gingival fibroblasts) cell line, and MRC-5 (Medical Research Council cell strain 5-human fibroblasts derived from lung tissue) cell line of the most potent compounds (compound $\mathbf{1}$ and $\mathbf{9}$ ) were also evaluated to show cytotoxic effects on healthy cells if existing.

\section{RESULTS AND DISCUSSION}

\subsection{Chemistry}

KA represents an attractive chemical structure of six-membered ring with one oxygen heteroatom carrying a hydroxy substituent at $5^{\text {th }}$ position which gives phenolic properties and a primer alcoholic hydroxy substituent at $2^{\text {nd }}$ position. Also KA is a good metal chelating agent that has capability to prevent oxidation and forms stable complexes with a broad range of different metal ions showing various type of biological activities. However, this hydrophilic property has restricted its application in cosmetic and pharmaceutical products. Therefore, KA has been studied intensively by several researchers in order to improve the chemical and biological activities.

In particular, chlorokojic acid (2-chloromethyl-5-hydroxy-4H-pyran-4-one, CKA) which is obtained by chlorination of the 2-hydroxymethyl moiety of KA using thionyl chloride at room temperature, is another important halogen derivative from the chemical view. It is a good ligand for the nucleophilic and electrophilic substitution reactions depending on the reagent type having antibacterial, antifungal and antiviral activities as well [13].

Mannich type reactions are multicomponent condensation reactions involving active hydrogen containing compound, formaline and a secondary amine. It has advantages ranging from lower reaction times, increased reaction rates to higher yields and reproducibility [20]. The amino alkylation of aromatic structures by Mannich reaction, is used for the synthesis and modification of biologically active compounds. Particularly, the incorporation of piperazine is commonly preferred due to its easy modifiability [21].

The drug candidates should be lipophilic enough to penetrate to lipid core of cell membranes, therefore to increase this penetration, some lipophilic groups are commonly substituted to gain better molecules in drug design. Moreover, the lipophilic properties of a compound could be adjusted through a Mannich reaction by using appropriate amine reagent. Therefore, increased cellular permeability could be accessed by the hydrophobic structure. This distinction is well used in designing anticancer agents with cytotoxic effects observing that they do not alkylate cellular hydroxyl and amino groups causing mutagenicity and genotoxicity [10]. In this study, it is observed that the lipophilicity of newly synthesized Mannich bases (clogP: 1.449-4.084) were significantly higher than the starting compound KA (clogP: -1.39) by using theoretical values obtained from ChemDraw Ultra 8.0.3.

KA and CKA have phenol-like properties facilitating their aminomethylation at room temperature in a short time with high yields, during the Mannich reaction ortho to the enolic hydroxyl group. The mechanism of this Mannich reaction relies on enhanced activity in a basic medium due to increased electronegativity at 
$6^{\text {th }}$-position of the hydroxypyrone scaffold [22]. Within the scope of the present study, twenty six Mannich bases comprising eleven novel compounds were synthesized using the methodology shown in Figure 1.

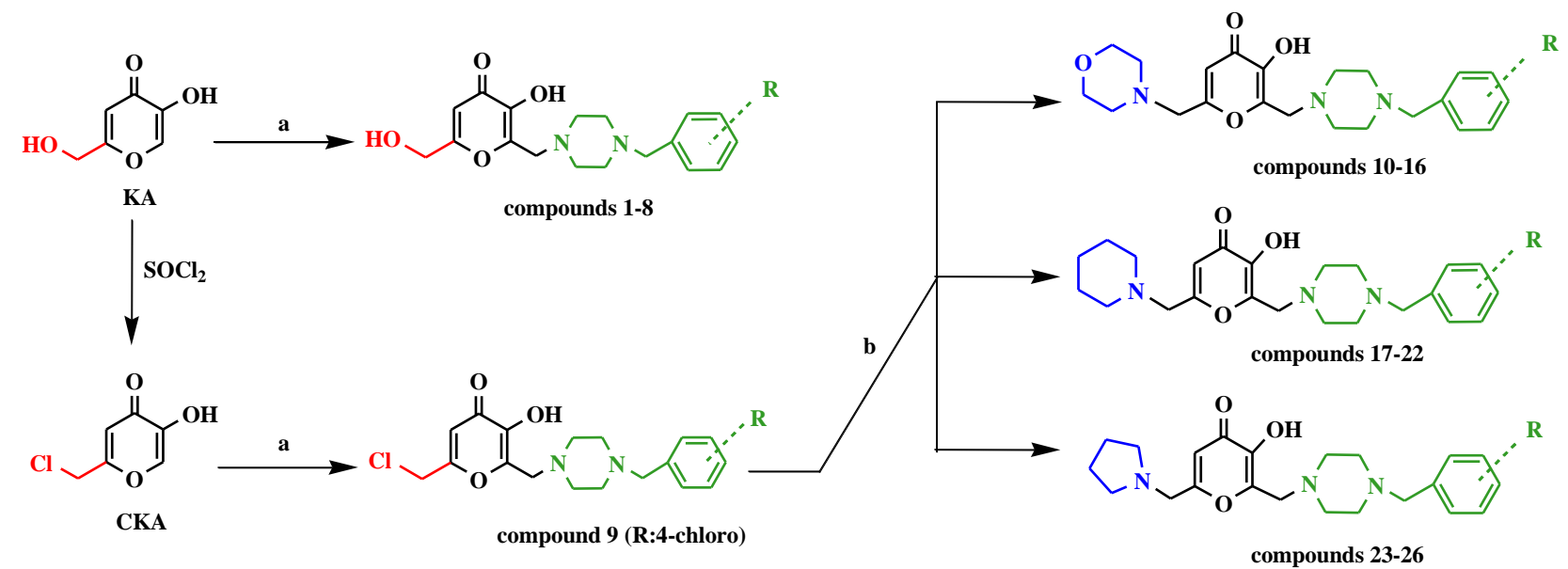

R: 3-chloro; 4-chloro; 3,4-dichloro; 2,6-dichloro 4-fluoro; 2,5-difluoro; 3-trifluoromethyl; 2-chloro-6-fluoro; 4-bromo-2-fluoro.

Figure 1. Synthesis of compounds 1-26; Reagents and conditions: (a: Method I) substituted benzyl piperazine derivatives, formaline $37 \%, \mathrm{MeOH}$, rt, (b: Method II) morpholine/piperidine/pyrrolidine, $\mathrm{K}_{2} \mathrm{CO}_{3}, \mathrm{DMF}$, $0^{\circ} \mathrm{C}$; KA: Kojic acid, CKA: Chlorokojic acid.

Mannich bases were prepared by a high yield reaction of appropriately substituted benzylpiperazine derivatives with KA or CKA and formaline at room temperature. The resulting precipitate was collected by filtration and washed with cold methanol. In the next step, 2-substituted-3-hydroxy-6(morpholinylmethyl/piperidinylmethyl/pyrrolidinylmethyl)-4H-pyran-4-one (compounds 10-26) were synthesized following the reaction of Mannich bases of CKA with morpholine, piperidine or pyrollidine moieties. Reaction occurred in the aprotic solvent DMF and basic medium provided by potassium carbonate. The reaction started at cold temperature and finished at room temperature after $36 \mathrm{~h}$. The final mixture extracted using dichloromethane. The organic phase was evaporated to dryness which were further purified by recrystallization in ethyl acetate or ethyl acetate/petroleum ether. Formation of the desired new Mannich bases was confirmed on the basis of elementary analysis and spectroscopic methods. Physical properties including their molecular formula, molecular weights, yields and melting points are given in monographs.

All the compounds presented herein were characterized by using FT-IR, ESI-MS, ${ }^{1} \mathrm{H}-$ and ${ }^{13} \mathrm{C}-\mathrm{NMR}$ spectroscopy. The selected diagnostic bands of IR spectra provided useful information for determining structures in terms of absorption bands that are about $1620 \mathrm{~cm}^{-1}$ due to $(\mathrm{C}=\mathrm{O})$ streching of pyranone ring. Due to the hydroxymethyl moiety showing intra- and intermolecular two hydrogen bondings, these signals were observed at lower frequencies. Formation of the Mannich bases was further confirmed with the ${ }^{1} \mathrm{H}-\mathrm{NMR}$ spectra. Assignment of the signals was based on the chemical shifts and intensity pattern. Most of the compounds exhibited broad singlet peaks due to nitrogen atoms of the piperazine and pyrollidine rings. The The ${ }^{13} \mathrm{C}-\mathrm{NMR}$ spectra of the compounds clearly provides the evidence of the synthesis of the proposed structures. Carbonyl carbons of the hydroxypyrone ring displayed signal at $173 \mathrm{ppm}$. Peaks around 51-55 ppm were due to piperazine carbon atoms. Also, in the electromass ionisaiton mass spectrums showed molecular ion peaks equivalent to their molecular formulae and characteristic $\mathrm{M}^{+}+23(\mathrm{Na})$ peaks and $\mathrm{M}^{+}+1$ isotope peaks owing to bromine and chlorine atoms with the ratio of 1:1 and 3:1, respectively.

\subsection{Assesment of the cytotoxicity of compounds on A375, HGF-1 and MRC-5 cell lines}

In this study, the impact of KA, CKA and their twenty six derivatives on the cell viability of A375 malignant melanoma cells was explored and compared with the efficacy of currently used drugs (dacarbazine, temozolomide and lenalidomide) in the treatment of malignant melanoma.

The effect of dacarbazine on melanoma cells is not prominent as shown in previous studies [23, 24]. IC 50 values of the cells for temozolomide and lenalidomide were even lower than dacarbazine suggesting that none of these FDA approved drugs were potentially effective on this cell line, particularly. 
Dacarbazine, temozolomide and lenalidomide were chosen as control agents among the commercially available drugs for the treatment of malignant melanoma. Doses were administered starting from $150 \mu \mathrm{M}$ and gradually diluted to $100,75,50,25,12.5$ and $6.25 \mu \mathrm{M}$. Cytotoxicity of all the synthesized compounds against A375 cell line which were well-studied malignant melanoma cells that carry the activating BRAF V600E mutation, have been assessed using SRB assay [25]. This test is based on the colorimetric measurement of cellular protein content. It is particularly the test of choice for large-scale drug-screening studies compared to other cytotoxicity tests, since it measures the cellular protein content- but not the electrochemical potential gradient- and provides accurate and reproducible results that are linearly correlated with viability. Therefore, National Cancer Institute indicated SRB test as the standard protocol for the high-volume compound screening. $\mathrm{IC}_{50}$ cytotoxicity values $(\mu \mathrm{M})$ of the compounds for A375 cells and structures as well as melting points are given in Table 1.

Table 1. Chemical structures, melting points and $\mathrm{IC}_{50}(\mu \mathrm{M})$ values of $\mathrm{A} 375$ cells exposed to the compounds and control agents, for $48 \mathrm{~h}\left(\mathrm{n}=3 ;{ }^{*} \mathrm{p}<0.05\right)$.<smiles>[R]CN1CCN(Cc2oc([R])cc(=O)c2O)CC1</smiles>

\begin{tabular}{|c|c|c|c|c|c|}
\hline $\begin{array}{c}\text { Comp. } \\
\text { no }\end{array}$ & $\mathbf{R}$ & $\mathbf{R}^{\prime}$ & $\begin{array}{l}\text { Mol. Formula } \\
\text { (Mol. Wt. g/mol) }\end{array}$ & $\begin{array}{c}\text { Melting point } \\
\left({ }^{\circ} \mathrm{C}\right)\end{array}$ & $\begin{array}{l}\mathrm{IC}_{50} \\
(\mu \mathrm{M})\end{array}$ \\
\hline $1^{a}$ & $-\mathrm{CH}_{2} \mathrm{OH}$ & & $\begin{array}{c}\mathrm{C}_{18} \mathrm{H}_{21} \mathrm{ClN}_{2} \mathrm{O}_{4} \\
(364.82)\end{array}$ & $170-1$ & $73.74 \pm 1.04$ \\
\hline $2^{\mathrm{a}}$ & $-\mathrm{CH}_{2} \mathrm{OH}$ & & $\begin{array}{c}\mathrm{C}_{18} \mathrm{H}_{21} \mathrm{ClN}_{2} \mathrm{O}_{4} \\
(364.82)\end{array}$ & Dec. & $78.01 \pm 1.02$ \\
\hline $3^{b}$ & $-\mathrm{CH}_{2} \mathrm{OH}$ & & $\begin{array}{c}\mathrm{C}_{18} \mathrm{H}_{20} \mathrm{Cl}_{2} \mathrm{~N}_{2} \mathrm{O}_{4} \\
(399.27)\end{array}$ & 196-7 & $84.5 \pm 1.04$ \\
\hline $4^{a}$ & $-\mathrm{CH}_{2} \mathrm{OH}$ & & $\begin{array}{c}\mathrm{C}_{18} \mathrm{H}_{21} \mathrm{FN}_{2} \mathrm{O}_{4} \\
(348.37)\end{array}$ & $187-8$ & $92.01 \pm 1.03$ \\
\hline $5^{a}$ & $-\mathrm{CH}_{2} \mathrm{OH}$ & & $\begin{array}{c}\mathrm{C}_{18} \mathrm{H}_{20} \mathrm{~F}_{2} \mathrm{~N}_{2} \mathrm{O}_{4} \\
(366.36)\end{array}$ & $176-7$ & $87.56 \pm 1.04$ \\
\hline 6 & $-\mathrm{CH}_{2} \mathrm{OH}$ & & $\begin{array}{c}\mathrm{C}_{19} \mathrm{H}_{21} \mathrm{~F}_{3} \mathrm{~N}_{2} \mathrm{O}_{4} \\
(398.38)\end{array}$ & $183-4$ & $89.87 \pm 1.04$ \\
\hline 7 & $-\mathrm{CH}_{2} \mathrm{OH}$ & & $\begin{array}{c}\mathrm{C}_{18} \mathrm{H}_{20} \mathrm{ClFN}_{2} \mathrm{O}_{4} \\
(382.81)\end{array}$ & $184-5$ & $95.59 \pm 1.04$ \\
\hline 8 & $-\mathrm{CH}_{2} \mathrm{OH}$ & & $\begin{array}{c}\mathrm{C}_{18} \mathrm{H}_{20} \mathrm{BrFN}_{2} \mathrm{O}_{4} \\
(427.26)\end{array}$ & Dec. & $77.33 \pm 1.11$ \\
\hline 9c & $-\mathrm{CH}_{2} \mathrm{Cl}$ & & $\begin{array}{c}\mathrm{C}_{18} \mathrm{H}_{20} \mathrm{Cl}_{2} \mathrm{~N}_{2} \mathrm{O}_{3} \\
(383.27)\end{array}$ & $168-9$ & $71.27 \pm 1.05$ \\
\hline $10^{a}$ & & & $\begin{array}{c}\mathrm{C}_{22} \mathrm{H}_{28} \mathrm{ClN}_{3} \mathrm{O}_{4} \\
(433.93)\end{array}$ & $137-8$ & $85.36 \pm 1.03$ \\
\hline $11^{a}$ & $\mathrm{CH}_{2}-$ & & $\begin{array}{c}\mathrm{C}_{22} \mathrm{H}_{27} \mathrm{Cl}_{2} \mathrm{~N}_{3} \mathrm{O}_{4} \\
(468.37)\end{array}$ & $142-4$ & $78.64 \pm 1.04$ \\
\hline
\end{tabular}




\begin{tabular}{|c|c|c|c|c|c|}
\hline $\begin{array}{c}\text { Comp. } \\
\text { no }\end{array}$ & $\mathbf{R}$ & $\mathbf{R}^{\prime}$ & $\begin{array}{l}\text { Mol. Formula } \\
\text { (Mol. Wt. g/mol) }\end{array}$ & $\begin{array}{c}\text { Melting point } \\
\left({ }^{\circ} \mathrm{C}\right)\end{array}$ & $\begin{array}{l}\mathrm{IC}_{50} \\
(\mu \mathrm{M})\end{array}$ \\
\hline 12 & & & $\begin{array}{c}\mathrm{C}_{22} \mathrm{H}_{27} \mathrm{Cl}_{2} \mathrm{~N}_{3} \mathrm{O}_{4} \\
(468.37)\end{array}$ & $158-9$ & $88.04 \pm 1.03$ \\
\hline $13^{a}$ & & & $\begin{array}{c}\mathrm{C}_{22} \mathrm{H}_{28} \mathrm{FN}_{3} \mathrm{O}_{4} \\
(417.47)\end{array}$ & $156-7$ & $81.97 \pm 1.05$ \\
\hline $14^{a}$ & & & $\begin{array}{c}\mathrm{C}_{22} \mathrm{H}_{27} \mathrm{~F}_{2} \mathrm{~N}_{3} \mathrm{O}_{4} \\
(435.46)\end{array}$ & $107-8$ & $92.53 \pm 1.03$ \\
\hline 15 & & & $\begin{array}{c}\mathrm{C}_{23} \mathrm{H}_{28} \mathrm{~F}_{3} \mathrm{~N}_{3} \mathrm{O}_{4} \\
(467.48)\end{array}$ & $142-4$ & $86.86 \pm 1.05$ \\
\hline 16 & & & $\begin{array}{c}\mathrm{C}_{22} \mathrm{H}_{27} \mathrm{ClFN}_{3} \mathrm{O}_{4} \\
(451.92)\end{array}$ & $144-5$ & $91.05 \pm 1.04$ \\
\hline $17^{a}$ & & & $\begin{array}{c}\mathrm{C}_{23} \mathrm{H}_{30} \mathrm{ClN}_{3} \mathrm{O}_{3} \\
(431.96)\end{array}$ & $145-7$ & $92.37 \pm 1.03$ \\
\hline 18 & & & $\begin{array}{c}\mathrm{C}_{23} \mathrm{H}_{29} \mathrm{Cl}_{2} \mathrm{~N}_{3} \mathrm{O}_{3} \\
(466.40)\end{array}$ & $160-1$ & $84.24 \pm 1.03$ \\
\hline $19^{a}$ & & & $\begin{array}{c}\mathrm{C}_{23} \mathrm{H}_{30} \mathrm{FN}_{3} \mathrm{O}_{3} \\
(401.47)\end{array}$ & $159-60$ & $89.55 \pm 1.13$ \\
\hline $20^{a}$ & & & $\begin{array}{c}\mathrm{C}_{23} \mathrm{H}_{29} \mathrm{~F}_{2} \mathrm{~N}_{3} \mathrm{O}_{3} \\
(433.49)\end{array}$ & $102-4$ & $93.7 \pm 1.02$ \\
\hline 21 & & & $\begin{array}{c}\mathrm{C}_{24} \mathrm{H}_{30} \mathrm{~F}_{3} \mathrm{~N}_{3} \mathrm{O}_{3} \\
(465.51)\end{array}$ & $156-7$ & $87.5 \pm 1.02$ \\
\hline 22 & & & $\begin{array}{c}\mathrm{C}_{23} \mathrm{H}_{29} \mathrm{ClFN}_{3} \mathrm{O}_{3} \\
(449.95)\end{array}$ & $140-2$ & $103 \pm 1.04$ \\
\hline $23^{a}$ & & & $\begin{array}{c}\mathrm{C}_{22} \mathrm{H}_{28} \mathrm{ClN}_{3} \mathrm{O}_{3} \\
417.93)\end{array}$ & $134-5$ & $95.9 \pm 1.02$ \\
\hline 24 & & & $\begin{array}{c}\mathrm{C}_{22} \mathrm{H}_{27} \mathrm{Cl}_{2} \mathrm{~N}_{3} \mathrm{O}_{3} \\
(452.37)\end{array}$ & $147-9$ & $87.45 \pm 1.02$ \\
\hline $25^{a}$ & & & $\begin{array}{c}\mathrm{C}_{22} \mathrm{H}_{27} \mathrm{~F}_{2} \mathrm{~N}_{3} \mathrm{O}_{3} \\
(419.46)\end{array}$ & $108-10$ & $91.42 \pm 1.03$ \\
\hline
\end{tabular}




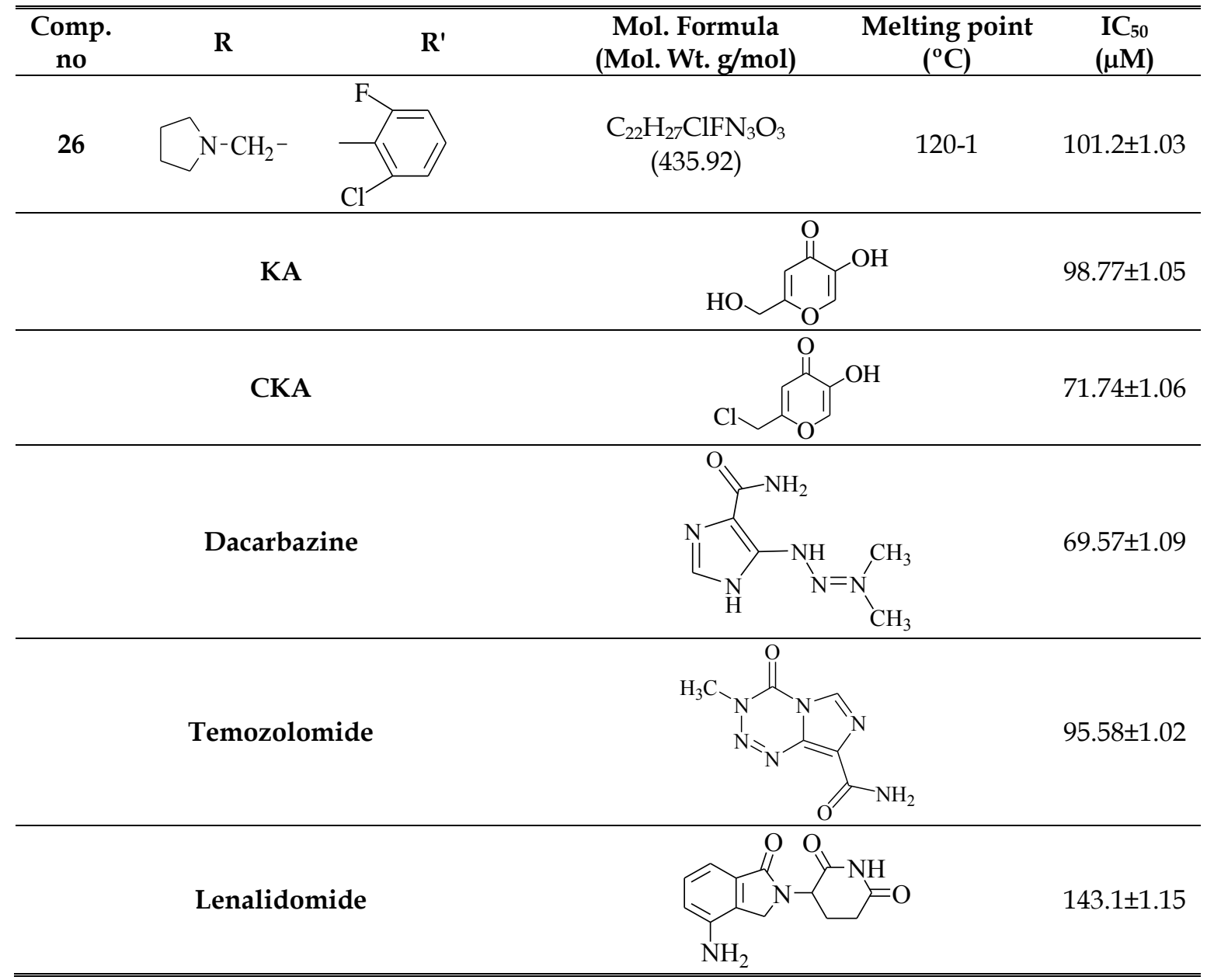

a [18],

$\mathrm{b}[17]$,

c [15],

Dec.: decomposed.

In this study, all of the twenty six Mannich bases carrying halogenic moieties were found to be cytototoxic against A375 cell line, possessing the V600E mutation which renders these melanoma cells very metastatic. With the exception of dacarbazine, twenty two of the compounds were found to have a more cytotoxic potential than FDA approved drugs temozolomide and lenalidomide. Also when compared with their initial molecules KA $\left(\mathrm{IC}_{50}: 98.77 \mu \mathrm{M}\right)$ and $\mathrm{CKA}\left(\mathrm{IC}_{50}: 71.74 \mu \mathrm{M}\right)$, the efficacy increased by gaining Mannich bases. Moreover, compound $\mathbf{1}\left(\mathrm{IC}_{50}: 73.74 \mu \mathrm{M}\right)$ and $9\left(\mathrm{IC}_{50}: 71.27 \mu \mathrm{M}\right)$ which have the most promising cytotoxic activities were also tested on healthy cell lines HGF- 1 and MRC- 5 cell lines. The concentration of the mentioned compounds needed for $50 \%$ cell growth inhibition were represented in Table 2 . The cytotoxic effects of these two compounds were very close to dacarbazine $\left(\mathrm{IC}_{50}: 69.57 \mu \mathrm{M}\right)$.

When it comes to the efficacy of a chemotherapeutical agent, showing no harmful effect on healthy cells is as much important as having cytotoxic potential on cancer cells. Having this in mind, it was shown that Compound 1 and 9 having approximately 10 and 4 fold higher IC $_{50}$ values for HGF- 1 and MRC- 5 cells than dacarbazine, respectively, indicating that these compounds were definitely less harmful than dacarbazine (Table 2). According to the results, when viewed from this aspect, it's very clear that the addition of Mannich bases improved the activity of the compounds with regard to KA and CKA.

Besides their cytotoxic effects, the impact on cellular morphology and proliferation of A375 cells was also investigated under light microscopy (Figure 2). Cellular structural integrity of the compounds 1 and 9 were found to be lost on microscopy in A375 cell line at $75 \mu \mathrm{M}$ doses which is the minimal dose among imaged doses that is higher than their $\mathrm{IC}_{50}$ values. This observation was recorded with the initial molecules (KA, CKA) and the control agents (dacarbazine, temozolomide, lenalidomide) at $100 \mu \mathrm{M}$ doses. 
Table 2. $\mathrm{IC}_{50}(\mu \mathrm{M})$ values of compound 1 and 9 against A375, HGF-1 and MRC-5 cells exposed to the compounds and control agents, for $48 \mathrm{~h}(\mathrm{n}=3$; * $\mathrm{p}<0.05)$.

\begin{tabular}{|c|c|c|c|c|}
\hline Name & Structure & A375 & HGF-1 & MRC-5 \\
\hline 9 & & $71.27 \pm 1.05$ & $2301 \pm 4.89$ & $\begin{array}{c}\text { No } \\
\text { inhibition }\end{array}$ \\
\hline 1 & & $73.74 \pm 1.04$ & $927.9 \pm 3.49$ & $\begin{array}{c}\text { No } \\
\text { inhibition }\end{array}$ \\
\hline & & $98.77 \pm 1.05$ & $193.9 \pm 1.30$ & $2123 \pm 14.9$ \\
\hline & & $71.74 \pm 1.06$ & $1958 \pm 3.88$ & $\begin{array}{c}\text { No } \\
\text { inhibition }\end{array}$ \\
\hline & azine & $69.57 \pm 1.09$ & $230.6 \pm 1.31$ & $230.3 \pm 1.22$ \\
\hline & omide & $95.58 \pm 1.02$ & $\begin{array}{c}\text { No } \\
\text { inhibition }\end{array}$ & $\begin{array}{c}\text { No } \\
\text { inhibition }\end{array}$ \\
\hline & omide & $143.1 \pm 1.15$ & $4353 \pm 4.07$ & $\begin{array}{c}\text { No } \\
\text { inhibition }\end{array}$ \\
\hline
\end{tabular}

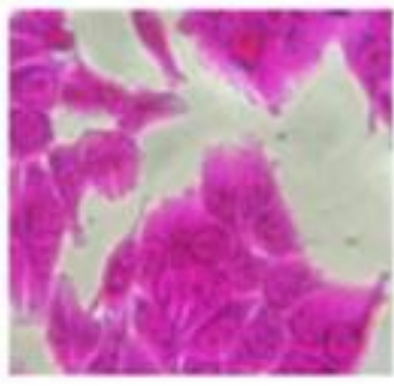

Compound $9(75 \mu \mathrm{M})$

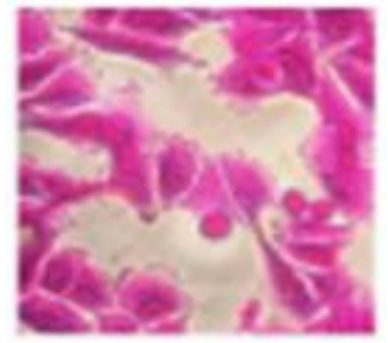

CKA $(100 \mu \mathrm{M})$

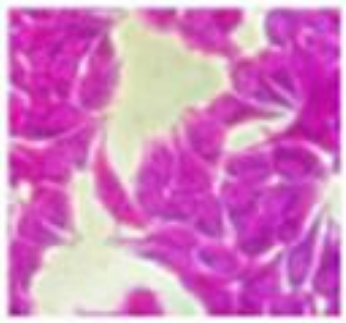

Dacarbazine $(100 \mu \mathrm{M})$

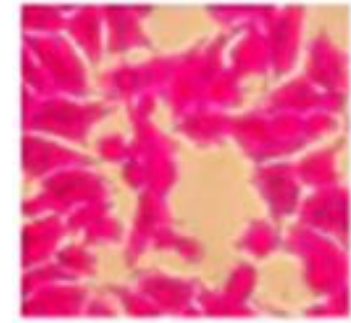

Temozolomide $(100 \mu \mathrm{M})$

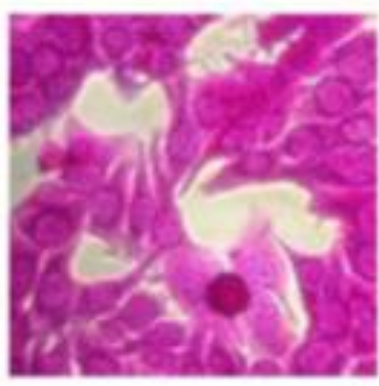

\section{Compound $1(75 \mu \mathrm{M})$}

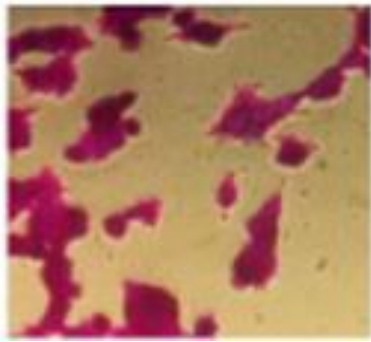

KA $(100 \mu \mathrm{M})$

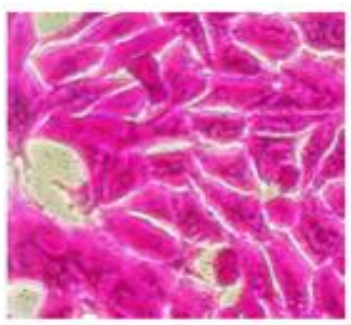

Lenalidomide $(100 \mu \mathrm{M})$

Figure 2. Morphological changes of $\mathrm{A} 375$ cells as response to exposure to $\mathrm{IC}_{50}$ concentrations of the compounds and control agents for $48 \mathrm{~h}$. 


\section{CONCLUSION}

In the present study, we reported the synthesis and cytotoxic evaluation of Mannich bases derived from KA against A375 human malignant melanoma cells. Compound $\mathbf{9}$ and $\mathbf{1}$ were identified as the most potent agents in the series having IC 50 values of $71.27 \mu \mathrm{M}$ and $73.74 \mu \mathrm{M}$, respectively. Since the basic principle of the chemotherapy is to inhibit the proliferation and growth of cancerogenic cells without damaging the patient's normal cells and tissues, these two compounds were tested on healthy cell lines HGF-1 and MRC-5 as well. They were detected to have higher $\mathrm{IC}_{50}$ values than dacarbazine commonly used drug in treatment of malignant melanoma, which means that these compounds were giving almost no harm to healthy cells. This study suggests further studies to understand the molecular mechanism of action by means of gaining anticancer agents those targeting in a large spectrum of malignant melanoma cells.

\section{MATERIALS AND METHODS}

\subsection{Chemistry}

All chemicals used herein were supplied by Merck (Darmstadt, Germany) and Aldrich Chemical Co. (Steinheim, Germany). Melting points were obtained on a Thomas Hoover Capillary Melting Point Apparatus (Philadelphia, PA, USA) and were uncorrected. IR spectra were recorded on a Perkin Elmer FT-IR 420 System, Spectrum BX spectrometer, Faculty of Pharmacy, Hacettepe University. ${ }^{1} \mathrm{H}$ and ${ }^{13} \mathrm{C}$ NMR spectra were carried out on a Varian Mercury $400 \mathrm{MHz}$ spectrophotometer in deutorochloroform $\left(\mathrm{CDCl}_{3}\right)$ and dimethylsulphoxyde $\left(\right.$ DMSO- $\left.\mathrm{d}_{6}\right)$. Tetramethylsilane (TMS) was used as an internal standard and chemical shifts were recorded as ppm coupling constants $(J)$ were given in Hz. Mass analysis was monitored with a Micromass ZQ LC-MS with Masslynx Software Version 4.1 by using electrospray ionization (ESI+) method and HPLC with Waters Alliance by using C18 columns. The elementary analyses were performed with a Leco CHNS-932 analyzer (Leco, St. Joseph, MI, USA) in the Central Laboratory of Ankara University, Faculty of Pharmacy. The purity of the compounds was assessed by thin layer chromatography (TLC) on Kieselgel $60 \mathrm{~F}_{254}$ (Merck, Darmstadt, Germany) chromatoplates. The used solvent system was chloroform:methanol:acetone (6:2:2).

\subsubsection{Synthesis of 3-hydroxy-6-chloromethyl/hydroxymethyl/methyl-2-substituted-4H-pyran-4-one derivatives (Method I)}

The secondary amine (substituted benzyl piperazine derivatives) and 37\% formaline were dissolved in $\mathrm{MeOH}$. KA or CKA was added to the solution and the mixture was stirred vigorously for 15 to $25 \mathrm{~min}$. The resulting precipitate was collected by filtration and washed with cold $\mathrm{MeOH}$. All crude products were recrystallized from the appropriate solvent.

2-((4-(3-trifluoromethylbenzyl)piperazin-1-yl)methyl)-3-hydroxy-6-(hydroxymethyl)-4H-pyran-4-one) (6)

$\mathrm{C}_{19} \mathrm{H}_{21} \mathrm{~F}_{3} \mathrm{~N}_{2} \mathrm{O}_{4}$ (M.W.: $398.38 \mathrm{~g} / \mathrm{mol}$ ), yield: 78\%, mp: 183-5 ${ }^{\circ} \mathrm{C}$, clog P: $1.476 . \%$ CHN Found (Calculated): C 57.12 (57.28), H 5.51 (5.31), N 7.14 (7.03). IR v (cm-1): 1609 (C=O), 1456 (C=C), 1198 (C-O). ${ }^{1}$ H-NMR (DMSO-d 400 $\mathrm{MHz}) \delta$ ppm 2.48-2.51 (8H, brs, piperazine- $H)$, $3.51\left(2 \mathrm{H}, \mathrm{s}\right.$, pyrane- $\mathrm{CH}_{2}$-piperazine), 3.55 (2H, s, $\left.-\mathrm{CH}_{2}-\mathrm{Ar}\right), 4.29$ $\left(2 \mathrm{H} ; \mathrm{s} ;-\mathrm{CH}_{2} \mathrm{OH}\right), 5.66\left(1 \mathrm{H} ;\right.$ brs; $\left.-\mathrm{CH}_{2} \mathrm{OH}\right) ; 6.32\left(1 \mathrm{H}, \mathrm{s}\right.$, pyrane- $\left.\mathrm{H}^{5}\right), 7.54-7.62(4 \mathrm{H} ; \mathrm{m} ; \mathrm{Ar}-\mathrm{H}),{ }^{13} \mathrm{C}-\mathrm{NMR} \delta$ (DMSO$\left.d_{6}, 100 \mathrm{MHz}\right) 52.32,53.47,59.56,59.50,60.96,108.85,122.86,125,129.03,132.71,139.75,143.56,146.44,167.49$, 173.49. ESI-MS (m/z) $399(100 \%, \mathrm{M}+\mathrm{H})^{+}, 421(\mathrm{M}+\mathrm{Na})^{+}$.

2-((4-(2-fluoro-6- chlorobenzyl)piperazin-1-yl)methyl)-3-hydroxy-6-(hydroxymethyl)-4H-pyran-4-one) (7)

$\mathrm{C}_{18} \mathrm{H}_{20} \mathrm{ClFN}_{2} \mathrm{O}_{4}$ (M.W.: $382.8 \mathrm{~g} / \mathrm{mol}$ ), yield: 85\%; mp: 184-5 ${ }^{\circ} \mathrm{C}$; clog P: 1.449. \% CHN Found (Calculated): C 56.07 (56.47), H 5.33 (5.27), N 7.40 (7.32). IR v (cm-1): 3274 (O-H), 2820 (C-H (aliphatic)), 1607 (C=O), 1454 (C=C), 1198 (C-O). ${ }^{1} \mathrm{H}-\mathrm{NMR}\left(\mathrm{DMSO}-d_{6}, 400 \mathrm{MHz}\right) \delta \mathrm{ppm}: 2.48-2.50$ (8H; m; piperazine); 3.47 (2H; s; pyrane-CH2piperazine); 3.56 (2H; s; - $\left.\mathrm{CH}_{2}-\mathrm{Ar}\right) ; 4.27$ (2H; s; $\left.-\mathrm{CH}_{2} \mathrm{OH}\right) ; 5.65\left(1 \mathrm{H} ; \mathrm{brs} ;-\mathrm{CH}_{2} \mathrm{OH}\right) ; 6.29\left(1 \mathrm{H}^{2}\right.$ s; $\left.\mathrm{H}^{5}\right) ; 7.16-7.37$ (3H; m; Ar-H). ESI-MS (m/z): $177(100 \%), 383(\mathrm{M}+\mathrm{H})^{+}, 385(\mathrm{M}+\mathrm{H}+2)^{+}, 405(\mathrm{M}+\mathrm{Na})^{+}, 407(\mathrm{M}+\mathrm{Na}+2)^{+}$.

\section{2-((4-(4-bromo-2-fluorobenzyl)piperazin-1-yl)methyl)-3-hydroxy-6-(hydroxymethyl)-4H-pyran-4-one (8)}

$\mathrm{C}_{18} \mathrm{H}_{20} \mathrm{BrFN}_{2} \mathrm{O}_{4} .0 .33 \mathrm{H}_{2} \mathrm{O}$ (M.W.: $433.21 \mathrm{~g} / \mathrm{mol}$ ), yield: 90\%; mp: decomposed; clog P: 1.599. \%CHN Found (Calculated): C 49.98 (49.91), H 4.75 (4.77), N 6.84 (6.47). IR v (cm-1): 3282 (O-H), 2825 (C-H (aliphatic)), 1608 (C=O), 1464 (C=C), 1089 (C-O). ${ }^{1} \mathrm{H}-\mathrm{NMR}$ (DMSO-d $\left.d_{6} 400 \mathrm{MHz}\right) \delta$ ppm: 2.37-2.51 (8H; m; piperazine), 3.47 (2H, s, $\left.-\mathrm{CH}_{2}-\right), 3.49\left(2 \mathrm{H}, \mathrm{s},-\mathrm{CH}_{2}-\mathrm{Ar}\right), 4.28\left(2 \mathrm{H}, \mathrm{s},-\mathrm{CH}_{2} \mathrm{OH}\right), 5.75\left(1 \mathrm{H}, \mathrm{brs},-\mathrm{CH}_{2} \mathrm{OH}\right), 6.30\left(1 \mathrm{H}, \mathrm{s}, \mathrm{H}^{5}\right), 7.32-7.49(3 \mathrm{H} ; \mathrm{m}$; Ar-H). ESI-MS (m/z): $177(100 \%), 427(\mathrm{M})^{+}, 449(\mathrm{M}+\mathrm{Na})^{+}$. 
4.1.2. Synthesis of 2-((4-(substituted benzyl)piperazine-1-yl)methyl)-3-hydroxy-6-

(morpholinyl/piperidinyl/pyrrolidinylmethyl)-4H-pyran-4-one derivatives (Method II)

Morpholine, piperidine or pyrrolidine were dissolved in dimethylformamide (DMF). Mannich base was added to solution in ice bath. $\mathrm{K}_{2} \mathrm{CO}_{3}$ was also added after mixing in magnetic stirrer. After $36 \mathrm{~h}$, the mixture was poured into iced water and extracted using organic solvents. After evaporating the organic phase, the resulting precipitate was washed with cold $\mathrm{MeOH}$ and recrystallized from the appropriate solvent.

2-((4-(2,6-dichlorobenzyl)piperazin-1-yl)methyl)-3-hydroxy-6-(morpholin-1-ylmethyl)-4H-pyran-4-one (12)

$\mathrm{C}_{22} \mathrm{H}_{27} \mathrm{Cl}_{2} \mathrm{~N}_{3} \mathrm{O}_{4}$. ${ }^{1 / 2} \quad \mathrm{H}_{2} \mathrm{O}$ (M.W.: $477.38 \mathrm{~g} / \mathrm{mol}$ ), yield: $14 \%$, mp: 158-9 ${ }^{\circ} \mathrm{C}$, clog P: 2.804. \%CHN Found (Calculated): C 55.61 (55.35), H 5.71 (5.91),N 9.19 (8.80). IR v (cm-1): 2813 (C-H (aliphatic)), 1621 (C=O), 1454, 1435 (C=C), 1194, 1114 (C-N), 1003 (C-O). ${ }^{1} \mathrm{H}-\mathrm{NMR}\left(\mathrm{CDCl}_{3}, 400 \mathrm{MHz}\right) \delta$ ppm: 2.51 (4H; t; morpholine-CH2-N); 2.60-2.63 (8H; brs; piperazine- $H$ ); 3.36 (2H; s; morpholine- $\mathrm{CH}_{2}$-pyrane); 3.64 (2H; s; pyrane-CH $\mathrm{H}_{2}$-piperazine); 3.70 (4H; t; J=4.4 Hz; morpholine- $\left.\mathrm{CH}_{2}-\mathrm{O}\right)$; 3.75 (2H; s; $\left.-\mathrm{CH}_{2}-\mathrm{Ar}\right) ; 6.45\left(1 \mathrm{H} ; \mathrm{s} ;\right.$ pyrane- $\left.\mathrm{H}^{5}\right) ; 7.12\left(1 \mathrm{H} ; \mathrm{t} ; \mathrm{Ar}-\mathrm{H}^{4}\right)$; 7,27 (2H; t; Ar- $\left.H^{3^{\prime}, 5^{\prime}}\right),{ }^{13} \mathrm{C}-\mathrm{NMR} \delta\left(\mathrm{CDCl}_{3}, 100 \mathrm{MHz}\right)$ 52.66, 53.00, 53.48, 55.61, 56.20, 66.79, 111.81, 128.35, 128.87, $134.08,136.94,143.95,145.34,164.33,173.80$. ESI-MS (m/z) $235(100 \%), 468(\mathrm{M})^{+}$.

\section{2-((4-(3-trifluoromethylbenzyl)piperazin-1-yl)methyl)-3-hydroxy-6-(morpholin-1-ylmethyl)-4H-pyran-4-one (15)}

$\mathrm{C}_{23} \mathrm{H}_{28} \mathrm{~F}_{3} \mathrm{~N}_{3} \mathrm{O}_{4}$ (M.W.: $467.48 \mathrm{~g} / \mathrm{mol}$ ), yield: $50 \%$, mp: 142- $4^{\circ} \mathrm{C}$, clog P: $2.261 . \%$ CHN Found (Calculated): C 58.83 (59.09), H 6.08 (6.04), N 9.06 (8.99). IR v (cm-1): 3293 (O-H), 2824 (C-H (aliphatic)), 1635 (C=O), 1462 (C=C), 1328 (C-N), 1117 (C-O). ${ }^{1} \mathrm{H}-\mathrm{NMR}\left(\mathrm{CDCl}_{3}, 400 \mathrm{MHz}\right) \delta$ ppm 2.38-2.44 (8H, brs, piperazine- $\left.\mathrm{H}\right), 2.48-2.51(4 \mathrm{H}, \mathrm{m}$, morpholine- $\left.\mathrm{CH}_{2}-\mathrm{N}\right), 3.39$ (2H, s, morpholine- $\mathrm{CH}_{2}$-pyrane), 3.54 (2H, s, pyrane- $\mathrm{CH}_{2}$-piperazine), 3.55 (2H, s, $\left.\mathrm{CH}_{2}-\mathrm{Ar}\right), 3.57\left(4 \mathrm{H}, \mathrm{m}\right.$, morpholine- $\left.\mathrm{CH}_{2}-\mathrm{O}\right), 6.34\left(1 \mathrm{H}, \mathrm{s}\right.$, pyrane- $\left.H^{5}\right), 7.52-7.60(4 \mathrm{H} ; \mathrm{m} ; \mathrm{Ar}-H),{ }^{13} \mathrm{C}-\mathrm{NMR} \delta\left(\mathrm{CDCl}_{3}\right.$, $100 \mathrm{MHz})$ 52.23, 52.39, 52.76, 53.31, 58.60, 61.00, 66.09, 111.83, 120.18, 122.89, 123.56, 123.60, 124.80, 124.84, $124.88,125.59,128.30,128.47,128.78,129.09,129.13,129.40,132.69,139.65,147.00 .163 .66,173.33$. ESI-MS (m/z) $468(100 \%, \mathrm{M})^{+}, 469(\mathrm{M}+2)^{+}$.

\section{2-((4-(2-fluoro-6-chlorobenzyl)piperazin-1-yl)methyl)-3-hydroxy-6-(morpholin-1-ylmethyl)-4H-pyran-4-one (16)}

$\mathrm{C}_{22} \mathrm{H}_{27} \mathrm{ClFN}_{3} \mathrm{O}_{4}$ (M.W.: $451.92 \mathrm{~g} / \mathrm{mol}$ ), yield: $30 \%$, mp: $144-5^{\circ} \mathrm{C}$, clog P: 2.234. \%CHN Found (Calculated): C 58.21 (58.47), H 6.17 (6.02), N 9.50 (9.30). IR v (cm-1): 2820 (C-H (aliphatic)), 1624 (C=O), 1453 (C=C), 1198 (CN), 1113 (C-O). ${ }^{1} \mathrm{H}-\mathrm{NMR}$ (DMSO- $\left.d_{6}, 400 \mathrm{MHz}\right) \delta \mathrm{ppm}: 2.40-2.41(8 \mathrm{H}$, brs, piperazine- $H$ ), $2.48(4 \mathrm{H}, \mathrm{m}$, morpholine- $\left.\mathrm{CH}_{2}-\mathrm{N}\right), 3.37\left(2 \mathrm{H}, \mathrm{s}\right.$, morpholine- $\mathrm{CH}_{2}$-pyrane), 3.49 (2H, s, pyrane- $\mathrm{CH}_{2}$-piperazine), 3.53 (4H, $\mathrm{t}$, morpholine- $\left.\mathrm{CH}_{2}-\mathrm{O}\right), 3.56\left(2 \mathrm{H}, \mathrm{s},-\mathrm{CH}_{2}-\mathrm{Ar}\right), 6.31\left(1 \mathrm{H}, \mathrm{s}\right.$, pyrane- $\left.H^{5}\right), 7.18\left(1 \mathrm{H}, \mathrm{t}, \mathrm{Ar}-\mathrm{H}^{3}\right), 7.28-7.37(2 \mathrm{H}, \mathrm{m}, \mathrm{Ar}-$ $\left.H^{4^{\prime}, 5^{\prime}}\right),{ }^{13} \mathrm{C}-\mathrm{NMR} \delta\left(\mathrm{DMSO}-d_{6}, 100 \mathrm{MHz}\right)$ 51.77, 52.11, 52.30, 52.71, 53.16, 58.52, 66.05, 111.77, 114.10, 114.33, $123.17,123.35,125.41,125.44,129.91,130.00,135.49,135.55,143.59,146.94,160.14,162.60,163.61,173.28$. ESIMS (m/z) $452(100 \%, M)^{+}$.

\section{2-((4-(2,6-dichlorobenzyl)piperazin-1-yl)methyl)-3-hydroxy-6-(piperidin-1-ylmethyl)-4H-pyran-4-one (18)}

$\mathrm{C}_{23} \mathrm{H}_{29} \mathrm{Cl}_{2} \mathrm{~N}_{3} \mathrm{O}_{3} .{ }_{1}^{1 / 4} \mathrm{CH}_{3} \mathrm{OH}$ (M.W.: $474.41 \mathrm{~g} / \mathrm{mol}$ ), yield: 33\%, mp: 160-1 ${ }^{\circ} \mathrm{C}$, clog P: 4.084. \%CHN Found (Calculated): C 58.72 (58.86), H 6.41 (6.59), N 9.17 (8.86). IR v (cm-1): 3064 (O-H), 2810 (C-H (aliphatic)), 1620 $(\mathrm{C}=\mathrm{O}), 1435$ (C=C), 1196 (C-N), 1006 (C-O). ${ }^{1} \mathrm{H}-\mathrm{NMR}\left(\mathrm{CDCl}_{3}, 400 \mathrm{MHz}\right) \delta$ ppm 1.40-1.44 (2H, m, piperidine ${ }^{4}-$ $H), 1.54-1.60\left(4 \mathrm{H}, \mathrm{m}\right.$, piperidine $\left.{ }^{3,5}-H\right), 2.42\left(4 \mathrm{H}, \mathrm{t}, J=5.2 \mathrm{~Hz}\right.$, piperidine $\left.{ }^{2,6}-H\right), 2.59-2.63(8 \mathrm{H}, \mathrm{m}$, piperazine $), 3.32$ ( $2 \mathrm{H}$, s, piperidine- $\mathrm{CH}_{2}$-pyrane), 3.63 (2H, s, pyrane- $\mathrm{CH}_{2}$-piperazine), 3.75 ( $2 \mathrm{H}, \mathrm{s},-\mathrm{CH}_{2}$-Ar), 6.43 (1H, s, pyrane$\left.H^{5}\right), 7.12\left(1 \mathrm{H}, \mathrm{t}, \mathrm{Ar}-\mathrm{H}^{4^{\prime}}\right), 7.27\left(2 \mathrm{H}, \mathrm{t}, \mathrm{Ar}-\mathrm{H}^{3^{\prime}, 5^{\prime}}\right){ }^{13} \mathrm{C}-\mathrm{NMR} \delta\left(\mathrm{CDCl}_{3}, 100 \mathrm{MHz}\right) 23.91,25.87,52.66,52.83,54.53,55.57$, 56.20, 60.24, 111.65, 128.33, 128.83, 134.14, 136.95, 143.85, 142.21, 165.38, 173.91. ESI-MS (m/z) 234 (100\%), 466 $(\mathrm{M}+\mathrm{H})^{+}, 468(\mathrm{M}+\mathrm{H}+2)^{+}$.

2-((4-(3-trifluoromethylbenzyl)piperazin-1-yl)methyl)-3-hydroxy-6-(piperidin-1-ylmethyl)-4H-pyran-4-one (21)

$\mathrm{C}_{24} \mathrm{H}_{30} \mathrm{~F}_{3} \mathrm{~N}_{3} \mathrm{O}_{3}$ (M.W.: $465.51 \mathrm{~g} / \mathrm{mol}$ ), yield: 45\%, mp: 156-7 C, clog P: 3.541. \% CHN Found (Calculated): C 61.80 (61.92), H 6.80 (6.50), N 9.19 (9.03). IR v (cm-1): 2949, 2808 (C-H (aliphatic)), 1618 (C=O), 1456 (C=C), 1195 (C$\mathrm{N}), 1117$ (C-O). ${ }^{1} \mathrm{H}-\mathrm{NMR}\left(\mathrm{CDCl}_{3}, 400 \mathrm{MHz}\right) \delta \mathrm{ppm}$ 1.41-1.46 (2H, m, piperidine $\left.{ }^{4}-\mathrm{H}\right), 1.56-1.61(4 \mathrm{H}, \mathrm{m}$, piperidine $3,5-H), 2.45\left(4 \mathrm{H}, \mathrm{t}, J=5 \mathrm{~Hz}\right.$, piperidine $\left.{ }^{2,6}-H\right), 2.52(4 \mathrm{H}$, brs, piperazine), 2.65 (4H, brs, piperazine), 3.34 ( $2 \mathrm{H}$, s, piperidine- $\mathrm{CH}_{2}$-pyrane), 3.56 (2H, s, pyrane- $\mathrm{CH}_{2}$-piperazine), 3.67 (2H, s, $-\mathrm{CH}_{2}$-Ar), 6.45 (1H, s, pyrane$\left.H^{5}\right), 7.41\left(1 \mathrm{H}, \mathrm{t}, \mathrm{Ar}-\mathrm{H}^{5^{\prime}}\right), 7.50\left(2 \mathrm{H}, \mathrm{d}, \mathrm{Ar}-\mathrm{H}^{4^{\prime}, 6^{\prime}}\right), 7.57\left(1 \mathrm{H} ; \mathrm{s} ; \mathrm{Ar}-\mathrm{H}^{2^{\prime}}\right) .{ }^{13} \mathrm{C}-\mathrm{NMR} \delta\left(\mathrm{CDCl}_{3}, 100 \mathrm{MHz}\right) 23.88,25.86$, 52.76, 54.52, 55.04, 60.22, 62.16, 111.30, 122.81, 123.97, 125.57, 130.56, 130.88, 132.26, 139.03, 143.77, 145.22, 165.67, 173.84. ESI-MS (m/z) $234(100 \%), 466(\mathrm{M}+\mathrm{H})^{+}$. 


\section{2-((4-(2-fluoro-6-chlorobenzyl)piperazin-1-yl)methyl)-3-hydroxy-6-(piperidin-1-ylmethyl)-4H-pyran-4-one (22)}

$\mathrm{C}_{23} \mathrm{H}_{29} \mathrm{ClFN}_{3} \mathrm{O}_{3}$ (M.W.: 449.95 g/mol), yield: $58 \%$, mp: 140-2 ${ }^{\circ} \mathrm{C}$, clog P: 3.514. \%CHN Found (Calculated): C 61.44 (61.40), H 6.83 (6.50), N 9.46 (9.34). IR v (cm-1): 3233 (O-H), 2811 (C-H (aliphatic)), 1620 (C=O), 1453 (C=C), 1197 (C-N), 1007 (C-O). ${ }^{1} \mathrm{H}-\mathrm{NMR}$ (DMSO- $\left.d_{6}, 400 \mathrm{MHz}\right) \delta \mathrm{ppm}: 1.34\left(2 \mathrm{H}, \mathrm{d}\right.$, piperidine $\left.{ }^{4}-H\right), 1.46(4 \mathrm{H}, \mathrm{t}$, piperidine $\left.{ }^{3,5}-H\right), 2.37\left(4 \mathrm{H}, \mathrm{t}\right.$, piperidine $\left.{ }^{2,6}-\mathrm{H}\right), 2.43(4 \mathrm{H}, \mathrm{brs}$, piperazine), $2.51(4 \mathrm{H}$, brs, piperazine), $3.35(2 \mathrm{H}, \mathrm{s}$, piperidine- $\mathrm{CH}_{2}$-pyrane), $3.52\left(2 \mathrm{H}, \mathrm{s}\right.$, pyrane $-\mathrm{CH}_{2}$-piperazine), $3.59\left(2 \mathrm{H}, \mathrm{s},-\mathrm{CH}_{2}-\mathrm{Ar}\right), 6.30\left(1 \mathrm{H}, \mathrm{s}\right.$, pyrane- $\left.\mathrm{H}^{5}\right)$, $7.20\left(1 \mathrm{H}, \mathrm{t}, \mathrm{Ar}-\mathrm{H}^{3^{\prime}}\right), 7.32-7.40\left(2 \mathrm{H}, \mathrm{m}, \mathrm{Ar}-\mathrm{H}^{4^{\prime}, 5^{\prime}}\right),{ }^{13} \mathrm{C}-\mathrm{NMR} \delta$ (DMSO- $\left.d_{6}, 100 \mathrm{MHz}\right) 23.55,25.47,51.76,52.10,52.28$, 53.12, 53.46, 59.00, 111.54, 114.08, 114.32, 123.13, 123.32, 125.42, 129.95, 135.54, 143.56, 146.83, 160.14, 162.61, 164.35, 173.37. ESI-MS (m/z) $226(100 \%), 450(\mathrm{M})^{+}$.

\section{2-((4-(2,6-dichlorobenzyl)piperazin-1-yl)methyl)-3-hydroxy-6-(pyrrolidin-1-ylmethyl)-4H-pyran-4-one (24)}

$\mathrm{C}_{22} \mathrm{H}_{27} \mathrm{Cl}_{2} \mathrm{~N}_{3} \mathrm{O}_{3} .3 / 4 \mathrm{H}_{2} \mathrm{O}$ (M.W.: $465.89 \mathrm{~g} / \mathrm{mol}$ ), yield: 68\%, mp: 147-9 ${ }^{\circ} \mathrm{C}$, clog P: 3.525. \%CHN Found (Calculated): C 56.77 (56.72), H 6.19 (6.17), N 9.29 (9.10). IR v (cm-1): 2952, 2825 (C-H (aliphatic)), 1634 (C=O), 1462 (C=C), 1199 (C-N), 1000 (C-O). ${ }^{1} \mathrm{H}-\mathrm{NMR}$ (DMSO-d $\left.{ }_{6}, 400 \mathrm{MHz}\right) \delta$ ppm 1.64-1.67 (4H, m, pyrrolidine $\left.{ }^{3,4}-H\right)$, 2.39-2.50 $\left(12 \mathrm{H}, \mathrm{brs}\right.$, pyrrolidine ${ }^{2,5}-\mathrm{H}$ and piperazine $), 3.45\left(2 \mathrm{H}, \mathrm{s}\right.$, pyrrolidine- $\mathrm{CH}_{2}$-pyrane), 3.49 (2H, s, pyrane$\mathrm{CH}_{2}$-piperazine), $3.63\left(2 \mathrm{H}, \mathrm{s},-\mathrm{CH}_{2}-\mathrm{Ar}\right), 6.27\left(1 \mathrm{H}, \mathrm{s}\right.$, pyrane- $\left.H^{5}\right), 7.28\left(1 \mathrm{H}, \mathrm{t}, \mathrm{Ar}-\mathrm{H}^{4}\right), 7.42\left(2 \mathrm{H}\right.$; t; Ar- $\left.\mathrm{H}^{3^{\prime}, 5^{\prime}}\right),{ }^{13} \mathrm{C}-$ NMR $\delta$ (DMSO- $d_{6}, 100 \mathrm{MHz}$ ) 23.20, 42.47, 52.25 , 53.14, 53.35, 55.77, 111.08, 128.47, 129.70, 133.57, 136.04, 143.59, 146.75, 164.89 ,173.44, ESI-MS (m/z) $227(100 \%), 453(\mathrm{M})^{+}, 455(\mathrm{M}+2)^{+}$.

\section{2-((4-(2-fluoro-6-chlorobenzyl)piperazin-1-yl)methyl)-3-hydroxy-6-(pyrrolidin-1-ylmethyl)-4H-pyran-4-one (26)}

$\mathrm{C}_{22} \mathrm{H}_{27} \mathrm{ClFN}_{3} \mathrm{O}_{3}$. ${ }^{1 / 2} \mathrm{CH}_{3} \mathrm{OH}$ (M.W.: $451.94 \mathrm{~g} / \mathrm{mol}$ ), yield: 35\%, mp: 120-1 ${ }^{\circ} \mathrm{C}$, clog P: 2.955. \%CHN Found (Calculated): C 60.08 (59.79), H 6.48 (6.47), N 9.61 (9.30). IR v (cm-1): 2942, 2814 (C-H (aliphatic)), 1620 (C=O), $1452(\mathrm{C}=\mathrm{C}), 1195(\mathrm{C}-\mathrm{N}), 1004(\mathrm{C}-\mathrm{O}) .{ }^{1} \mathrm{H}-\mathrm{NMR}$ (DMSO-d $\left.d_{6}, 400 \mathrm{MHz}\right) \delta$ ppm: 1.66-1.69 (4H, m, pyrrolidine ${ }^{3,4_{-}}$ $H), 2.43(4 \mathrm{H}$, brs, pyrrolidine $2,5-\mathrm{H}), 2.51\left(8 \mathrm{H}, \mathrm{m}\right.$, piperazine), $3.49\left(2 \mathrm{H}, \mathrm{s}\right.$, pyrrolidine- $\mathrm{CH}_{2}$-pyrane), $3.50(2 \mathrm{H}, \mathrm{s}$, pyrane- $\mathrm{CH}_{2}$-piperazine), $3.58\left(2 \mathrm{H}, \mathrm{s},-\mathrm{CH}_{2}-\mathrm{Ar}\right), 6.30\left(1 \mathrm{H}, \mathrm{s}\right.$, pyrane- $\left.H^{5}\right), 7.18-7.23\left(1 \mathrm{H}, \mathrm{m}, \mathrm{Ar}-H^{3^{\prime}}\right), 7.31-7.40(2 \mathrm{H}$; $\mathrm{m} ; \mathrm{Ar}-H^{4^{\prime}, 5^{\prime}}$ ), ${ }^{13} \mathrm{C}-\mathrm{NMR} \delta$ (DMSO- $d_{6}, 100 \mathrm{MHz}$ ) 23.19, 51.83, 52.28, 53.18, 53.32, 55.77, 111.13, 114.18, 114.41, $123.22,123.41,125.49,125.52,129.98,130.08,135.57,135.63,143.62,146.80,160.21,162.67,164.95,173.49$, ESIMS (m/z) $219(100 \%), 436(\mathrm{M})^{+}$.

\subsection{Biochemistry}

\subsubsection{Cell culture}

A375 human malignant melanoma (CRL-1619 ${ }^{\mathrm{TM}}$ ), HGF-1 human normal gingival biopsy (CRL-2014 ${ }^{\mathrm{TM}}$ ) and MRC-5 human normal lung (CRL-171 $\left.{ }^{\mathrm{TM}}\right)$ cell lines were purchased from ATCC. Cells were grown in DMEM supplemented with 10\% fetal bovine serum, 1\% $200 \mathrm{mM} \mathrm{L-glutamine,} 100 \mathrm{U} / \mathrm{mL}$ penicillin and 100 $\mu \mathrm{g} / \mathrm{mL}$ streptomycin. Cells were retained in $37^{\circ} \mathrm{C}$ incubator with $5 \% \mathrm{CO}_{2}$. Morphological properties and proliferation of the cells were observed with the aid of light microscope (NIKON Eclipse TS100).

\subsubsection{Cytotoxicity screening of compounds}

In order to determine the cytotoxic efficiency of the compounds, SRB assay was performed. The cells were seeded in 96-well plate with the concentration of $5 \times 10^{3}$ cells/well. At $80 \%$ confluency, cells were exposed to $6.25-150 \mu \mathrm{M}$ of the compounds to induce cytotoxicity. Subsequent to $48 \mathrm{~h}$ of incubation, cells were treated with $10 \%(\mathrm{w} / \mathrm{v})$ trichloroacetic acid (TCA) for $1 \mathrm{~h}$ at $4^{\circ} \mathrm{C}$. Cells were then washed with $\mathrm{dH}_{2} \mathrm{O}$ and $0.06 \%(\mathrm{w} / \mathrm{v})$ SRB (Santa Cruz Biotechnology, USA) was applied to each well and cells were incubated at room temperature for $30 \mathrm{~min}$. Subsequently, cells were treated with $1 \%(\mathrm{v} / \mathrm{v})$ acetic acid to remove excess dye. Finally, $200 \mu \mathrm{L}$ Tris base (10 mM, pH 10.5) (Sigma Aldrich, USA) was added to solubilize the dye. Optical density (OD) of the wells was determined at $510 \mathrm{~nm}$ through spectrophotometric microplate reader (PowerWave XS, Biotek, USA). Cells not treated with any derivatives were considered as control and to be $100 \%$ viable. The percentage of OD values compared to those of controls was assessed to determine the changes in viability.

\subsubsection{Statistical analysis}

GraphPad Prism 5.03 software was used for all statistical analyses. Student's t-test was used to compare all data to their related control. All $p$ values were based on the two-sided statistical analysis and $p<0.05$ was considered to be statistically significant. The $\%$ inhibition was calculated by substracting the values obtained by DMSO addition. Increasing concentrations of each compound $(6.5-2000 \mu \mathrm{M})$ were tested in triplicates and the $\mathrm{IC}_{50}$ values were assessed by constructing a non-linear regression curve. All experiments were carried out 
in at least triplicate $(\mathrm{n}=3)$. The logarithm of the inhibitor concentration (in $\mu \mathrm{M})$ was plotted against cell viability values (given as percentage).

Acknowledgements: This study was supported by The Scientific and Technological Research Council of Turkey (TÜBİTAK). (Project number: 111S311, 1135527 and 315S067)

Author contributions: Concept - G.K., A.E., M.D.A.; Design - G.K., A.E., M.D.A.; Supervision - G.K., A.E., M.D.A.; Resources - A.E., M.D.A.; Materials - G.K., A.E., M.D.A.; Data Collection and/or Processing - G.K., A.E., S.Ö., M.D.A.; Analysis and/or Interpretation - G.K., A.E., S.Ö., M.D.A.; Literature Search - G.K., A.E., S.Ö., M.D.A.; Writing Manuscript - G.K., A.E., S.Ö., M.D.A.; Critical Reviews - G.K., A.E., S.Ö., M.D.A.

Conflict of interest statement: The authors declared no conflict of interest.

\section{REFERENCES}

[1] Veselinovic JB, Veselinovic AM, Ilic-Tomic T, Davis R, O'Connor K, Pavic A, Nikodinovic-Runic J. Potent antimelanogenic activity and favorable toxicity profile of selected 4-phenyl hydroxycoumarins in the zebrafish model and the computational molecular modeling studies. Bioorg Med Chem. 2017; 25(24): 6286-6296. [CrossRef]

[2] McCourt C, Dolan O, Gormley G. Malignant Melanoma: A Pictorial Review. Ulster Med J. 2014; 83(2): $103-110$.

[3] Eugeniu B, Andreea Z, Serban A, Rares B, Liliana R, Codrina BA. Dynamic Changes of Molecular Markers during Natural History in Metastatic Melanoma: Ethical Issues and Lessons to Learn. J Integr Oncol. 2016; 5(3): E1000176. [CrossRef]

[4] Hanaizi Z, van Zwieten-Boot B, Calvo G, Lopez AS, van Dartel M, Camarero J, Abadie E, Pignatti F. The European Medicines Agency review of ipilimumab (Yervoy) for the treatment of advanced (unresectable or metastatic) melanoma in adults who have received prior therapy: summary of the scientific assessment of the Committee for Medicinal Products for Human Use. Eur J Cancer. 2012; 48(2): 237-242. [CrossRef]

[5] da Rocha Dias S, Salmonson T, van Zwieten-Boot B, Jonsson B, Marchetti S, Schellens JH, Giuliani R, Pignatti F. The European Medicines Agency review of vemurafenib (Zelboraf) for the treatment of adult patients with BRAF V600 mutation-positive unresectable or metastatic melanoma: summary of the scientific assessment of the Committee for Medicinal Products for Human Use. Eur J Cancer. 2013; 49(7): 1654-1661. [CrossRef]

[6] Bommareddy PK, Patel A, Hossain S, Kaufman HL, Talimogene laherparepvec (T-VEC) and other oncolytic viruses for the treatment of melanoma. Am J Clin Dermatol. 2017; 18(1): 1-15. [CrossRef]

[7] Mann T, Gervat W, Batzer J, Eggers K, Scherner C, Wenck H, Stab F, Hearing VJ, Röhm K-H, Kolbe L. Inhibition of Human Tyrosinase Requires Molecular Motifs Distinctively Different from Mushroom Tyrosinase. J Invest Dermatol. 2018; 138(7): 1601-1608. [CrossRef]

[8] Parvez S, Kang M, Chung H-S, Bae H. Naturally Occurring Tyrosinase Inhibitors: Mechanism and Applications in Skin Health, Cosmetics and Agriculture Industries. Phytother Res. 2007; 21: 805-816. [CrossRef]

[9] Sharma KV, Davids LM. Depigmentation in melanomas increases the efficacy of hypericin-mediated photodynamicinduced cell death. Photodiagnosis Photodyn Ther. 2012; 9: 156-163. [CrossRef]

[10] Tugrak M, Gul H, Sakagami H. Synthesis and cytotoxicities of 2-[4-hydroxy-(3,5-bis-aminomethyl)-benzylidene]indan-1-ones. Lett Drug Des Discov. 2015; 12: 806-812. [CrossRef]

[11] Aytemir, M.D., Karakaya, G. Kojic acid derivatives. In: Medicinal Chemistry and Drug Design; Deniz Ekinci, Ed.; InTech; Rijeka, 2012; Chapter 1, pp. 1-26.

[12] Aytemir MD, Calis U. Anticonvulsant and neurotoxicity evaluation of some novel kojic acids and allomaltol derivatives. Arch Pharm. 2010; 343(3): 173-181. [CrossRef]

[13] Aytemir MD, Ozcelik B. A study of cytotoxicity of novel chlorokojic acid derivatives with their antimicrobial and antiviral activities. Eur J Med Chem. 2010; 45(9): 4089-4095. [CrossRef]

[14] Aytemir MD, Ozcelik B. Synthesis and biological activities of new Mannich bases of chlorokojic acid derivatives. Med Chem Res. 2011; 20(4): 443-452. [CrossRef]

[15] Karakaya G, Aytemir MD, Ozcelik B, Calis U. Design, synthesis and in vivo/in vitro screening of novel chlorokojic acid derivatives. J Enz Inh Med Chem. 2013; 28(3): 627-638. [CrossRef]

[16] Aytemir MD, Ozcelik B, Karakaya G. Evaluation of bioactivities of chlorokojic acid derivatives against dermatophytes couplet with cytotoxicity. Bioorg Med Chem Lett. 2013; 23(12): 3646-3649. [CrossRef] 
[17] Aytemir MD, Özçelik B, Erdoğan İ. Orhan, Karakaya G, Şenol FS. Kojic acid-derived mannich bases with biological effect US 9,975,884 B2, May 22, 2018.

[18] Karakaya G, Türe A, Ercan A, Öncül S, Aytemir MD. Synthesis, Computational Molecular Docking Analysis and Effectiveness on Tyrosinase Inhibition of Kojic Acid Derivatives. Bioorg Chem. 2019; 88, 102950. [CrossRef]

[19] Karakaya G, Ercan A, Oncul S, Aytemir MD. Synthesis and cytotoxic evaluation of kojic acid derivatives with inhibitory activity on melanogenesis in human melanoma cells, Anticancer Agents Med Chem. 2018; 18: 2137-2148. [CrossRef]

[20] Ashok M, Holla BS, Poojary B. Convenient one pot synthesis and antimicrobial evaluation of some new Mannich bases carrying 4-methylthiobenzyl moiety. Eur J Med Chem. 2007; 42: 1095-1101. [CrossRef]

[21] Liu X, Xia W, Jiang Q, Yu P, Yue L. Chitosan oligosaccharide-N-chlorokojic acid mannich base polymer as a potential antibacterial material. Carbohydr Polym. 2018; 182: 225-234. [CrossRef]

[22] Ichimoto I, Ueda H, Tatsumi C, Fujii K, Sekido F, Nonomura S. Studies on Kojic Acid and its Related $\gamma$-Pyrone Compounds: Part VII. The Alkylation of kojic acid and pyromeconic acid through their Mannich base (Synthesis of Maltol-(1)) Part VIII. Synthesis of comenic acid from kojic acid (Synthesis of Maltol (2)). Agric Biol Chem. 1965; 29(2): 94-103. [CrossRef]

[23] Kim G, McKee AE, Ning YM, Hazarika M, Theoret M, Johnson JR, Xu QC, Tang S, Sridhara R, Jiang X, He K, Roscoe D, McGuinn WD, Helms WS, Russel AM, Miksinski SP, Zirkelbach JF, Earp J, Liu Q, Ibrahim A, Justice R, Pazdur R. FDA approval summary: vemurafenib for treatment of unresectable or metastatic melanoma with the BRAFV600E mutation. Clin Cancer Res. 2014; 20(19): 4994-5000. [CrossRef]

[24] Chapman PB, Robert C, Larkin J, Haanen JB, Ribas A, Hogg D, Hamid O, Ascierto PA, Testori A, Lorigan PC, Dummer R, Sosman JA, Flaherty KT, Chang I, Coleman S, Caro I, Hauschild A, McArthur GA. Vemurafenib in patients with BRAFV600 mutation-positive metastatic melanoma: final overall survival results of the randomized BRIM-3 study. Ann Oncol. 2017; 28(10): 2581-2587. [CrossRef]

[25] Vichai V, Kirtikara K, Sulforhodamine B colorimetric assay for cytotoxicity screening. Nat Protoc 2006; 1: 1112-1116. [CrossRef]

This is an open access article which is publicly available on our journal's website under Institutional Repository at http://dspace.marmara.edu.tr. 